\title{
Binary Continuous Image Decomposition for Multi-view Display
}

\author{
Gou Koutaki * \\ Kumamoto University
}
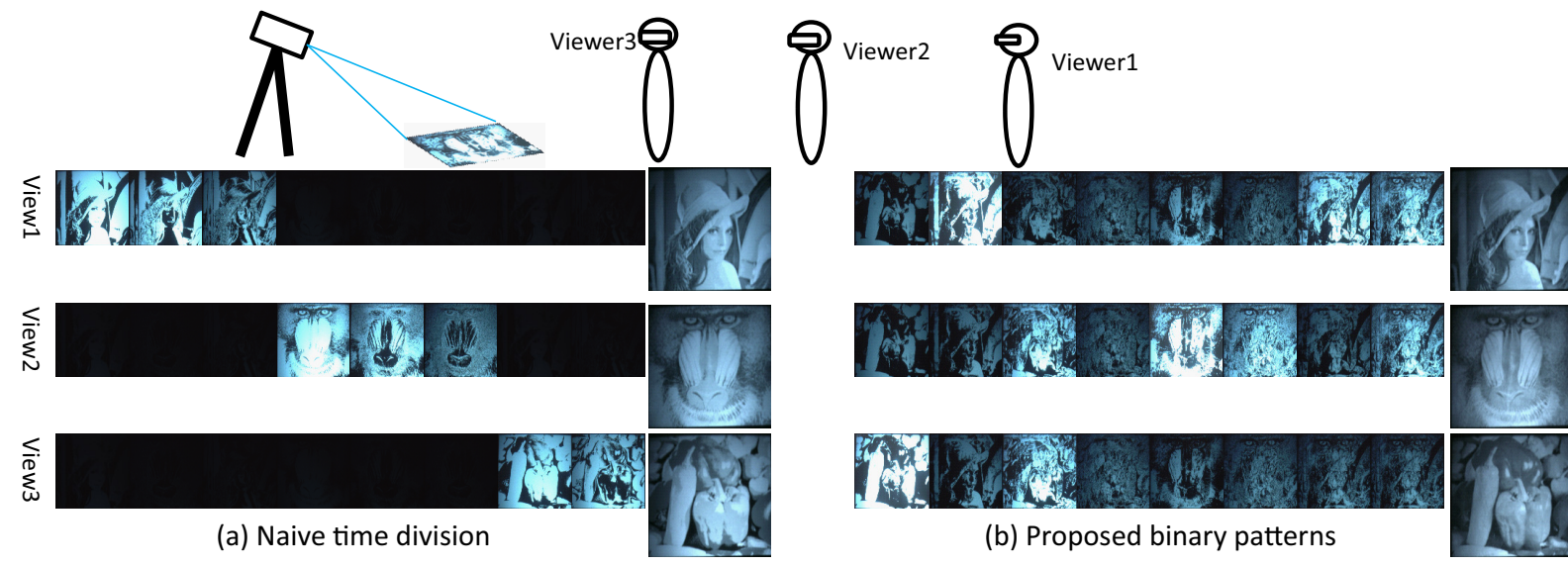

(b) Proposed binary patterns

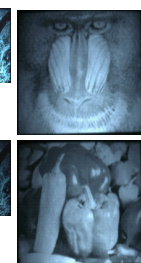

Figure 1: Three-view projection through active shutter glasses. (a) Conventional 0-1 active shutter with naive time division. (b) Proposed continuous transmittance active shutter. The conventional naive method projects many "black" frames, which cause flicker. The proposed method can reduce quantization errors, and causes less flicker than the naive time-division active shutter.

\begin{abstract}
This paper proposes multi-view display using a digital light processing (DLP) projector and new active shutter glasses. In conventional stereoscopic active shutter systems, active shutter glasses have a 0-1 (open and closed) state, and the right and left frames are temporally divided. However, this causes the display to flicker because the human eye perceives the appearance of black frames when the other shutter is closing. Furthermore, it is difficult to increase the number of views because the number of frames representing images is also divided. We solve these problems by extending the active shutter beyond the use of the $0-1$ state to a continuous range of states $[0,1]$ instead. This relaxation leads to the formulation of a new DLP imaging model and an optimization problem. The special structure of DLP binary imaging and the continuous transmittance of the new active shutter glasses require the solution of a binary continuous image decomposition problem. Although it contains NP-hard problems, the proposed algorithm can efficiently solve the problem. The implementation of our imaging system requires the development of an active shutter device with continuous transmittance. We implemented the control of the transmittance of the liquid crystal display (LCD) shutter by using a pulse-width modulation (PWM). A simulation and the developed multi-view display system were used to show that our model can represent multi-view images more accurately than the conventional time-division $0-1$ active shutter system.
\end{abstract}

Keywords: DLP, stereoscopic, 3D display, active shutter

Concepts: •Computing methodologies $\rightarrow$ Image processing;

*e-mail:koutaki@cs.kumamoto-u.ac.jp

Permission to make digital or hard copies of all or part of this work for personal or classroom use is granted without fee provided that copies are not made or distributed for profit or commercial advantage and that copies bear this notice and the full citation on the first page. Copyrights for components of this work owned by others than ACM must be honored. Abstracting with credit is permitted. To copy otherwise, or republish, to post on servers or to redistribute to lists, requires prior specific permission and/or a fee. Request

\section{Introduction}

Recently developed three-dimensional (3D) stereoscopic and additional multi-view displays are widely used in consumer electronics nowadays due to the evolution of high frame-rate displays and projectors and synchronized active shutter glasses. Stereoscopic displays are used in many types of entertainment content, such as 3D movies, 3D theater, and TV games or Virtual Reality applications.

Especially in recent years, the digital light processing (DLP) projector has become a standard instrument for 3D displays due to its merits in comparison with the conventional liquid crystal display (LCD) and projector. Because digital mirror devices (DMDs), which are micro-electro-mechanical systems (MEMS), can be manufactured in DLP by a complementary metal-oxide-semiconductor (CMOS) process, the cost of fabricating DMDs is low.

Figure 2 shows the mechanism of DLP imaging [Gove 1994][Hornbeck 1995]. DLP employs many micro-mirror arrays, which consist of millions of mirrors (digital micro-mirror devices, or DMDs), each approximately $10 \mu \mathrm{m}$ in size. The number of DMDs in a typical DLP projector is $1280 \times 800$ or $1920 \times 1024$. DLP can control each DMD to tilt at a very rapid speed, i.e., approximately 250 $\mu s$, where each DMD has an on-off state. The light emitted from the lamp or LED in the projector is reflected by the on-state of the $\mathrm{DMD}$, and the monocolor binary pattern can be projected on the screen through an optical lens unit. Although the DMD can only project a binary pattern, the rapid projection of many binary patterns in succession with different light intensities and colors, such as RGB, grayscale, or full color, results in images that can be seen by the human eye. For example, the projection of a $60-\mathrm{fps}, 24-$ bit full-color image is realized by projecting $60 \times 24=1440 \mathrm{~Hz}$ patterns per second.

Active shutter stereoscopic display can be enabled by taking ad-

permissions from permissions@acm.org. (c) 2016 ACM

SIGGRAPH '16 Technical Paper,, July 24-28, 2016, Anaheim, CA,

ISBN: 978-1-4503-4279-7/16/07

DOI: http://dx.doi.org/10.1145/2897824.2925949

ACM Trans. Graph., Vol. 35, No. 4, Article 69, Publication Date: July 2016 


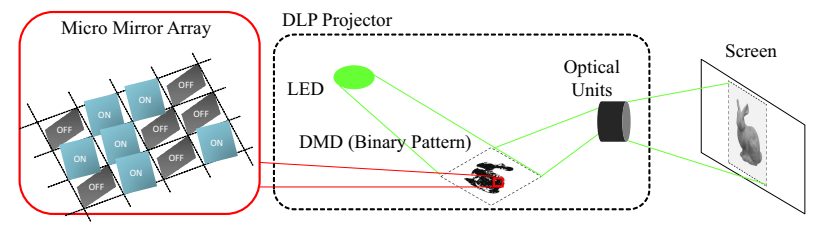

Figure 2: DLP projection structure. The light from LEDs is reflected by the DMD, and the binary patterns are projected onto a screen. The binary patterns are switched rapidly to display a grayscale or full-color image to the human eye.

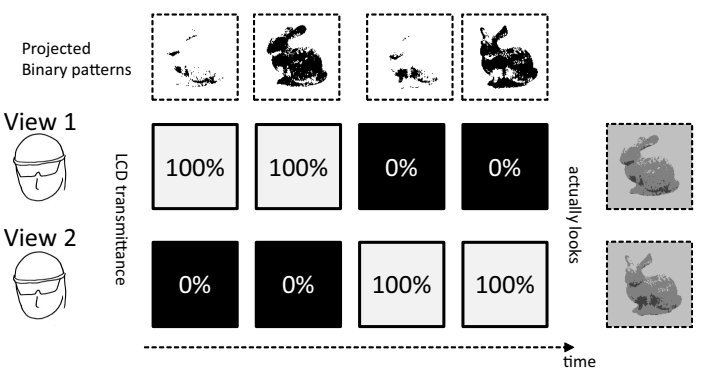

(a) Conventional active shutter

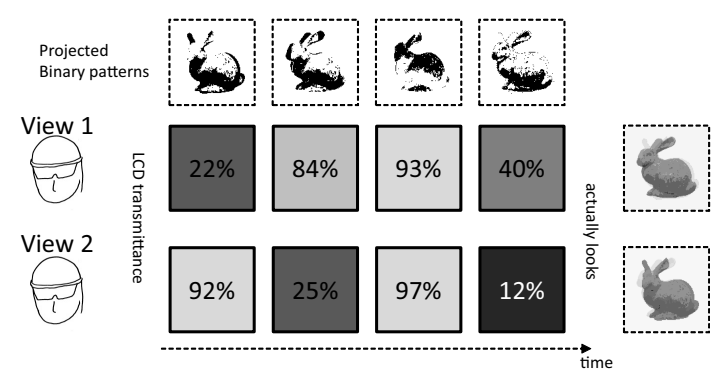

(b) Proposed active shutter

Figure 3: Comparison between the conventional and the proposed active shutter: (a) Conventional active shutter takes 0-1 (open and closed) states. (b) Proposed active shutter can take continuous transmittance in the range $[0,1]$.

vantage of the rapidness of DLP. The standard DLP stereoscopic method is based on the time-division method. Figure 3 (a) illustrates this method. Left-eye (View1) and right-eye (View2) images are projected alternately. When projecting the View1 pattern, the LCD shutter of View1 is open and that of View2 is closed. When projecting the View2 pattern, the opposite situation is implemented. The number of views can be increased by increasing the number of time divisions in the same way.

However, the active shutter stereoscopic system has the following limitations:

- Flicker due to "black" frames: The images of the right and left eyes are temporally divided. When we see the right-eye image, a "black" frame is seen by the left eye because the LCD shutter on the left is closed. This causes flicker. Simply increasing the number of views is problematic because this produces an even larger number of these "black" frames.

- Quantization errors: Increasing the number of views decreases the number of binary patterns per view. For example, subjecting four views for eight binary patterns to time division only results in a two-bit depth (four gray values) per view.

\subsection{Contributions}

We propose to address the above limitations by developing an active shutter system where the LCD shutter does not have a 0-1 state (open/closed), but a continuous intermediate state with a range of $[0,1]$ instead. Figure 3 (b) shows the proposed model. In this model, all binary patterns are visible to the human eye, and it avoids extreme "black" frames. Furthermore, it increases the number of binary patterns per view, and can reduce quantization errors.

By introducing this relaxation, the multi-view DLP imaging model is re-formulated. As a result, a binary continuous image decomposition problem emerges. However, this problem remains very difficult to solve exactly because it contains an NP-hard problem. Thus, in this study, we propose an efficient solver to achieve a realistic computational time.

To realize a continuous LCD shutter, we develop a pulse-width modulation (PWM) controllable LCD shutter and prove the feasibility of our model by using an actual projector system.

\section{Related work}

Many past research efforts have been devoted to stereoscopic and multi-view display using an active shutter.

\subsection{Active stereoscopic shutter}

The principle underlying the stereoscopic active shutter system was proposed in 1922. In the original 3D teleview system, which was developed by Laurens Hammond [Hammond 1922], films for the right and left eyes were projected to the screen alternately, and the mechanical active shutter scope was synchronized with the projector shutters. In recent decades, LCD glasses have been developed, through which the state of the shutter, open or closed, can be controlled by electrical voltage. The glasses are equipped with two LCD panels the polarization of which is altered by applying voltage. In this way, the polarization of the LCD is changed, thereby preventing light from entering; thus, the electric shutter closes. The LCD active shutter stereoscopic system was invented by Stephen McAllister in the mid-1970s, following which the 3D-VHS video system was developed in the mid-1980s [Peddie 2013].

\subsection{Stereoscopic display using DLP}

Since 2007, 3D-DLP technology has been widely implemented in consumer electronics [Urey et al. 2011]. Many companies have developed standard specifications for the 3D-DLP protocol and 3D glasses, such as DLP link, XpanD 3D, and Nvidia 3D Vision [Peddie 2013], which are synchronized to a DLP projector using infrared (IR) light, visible light, or radio frequency (RF) communication. For example, DLP is driven by $120 \mathrm{~Hz}$ or $144 \mathrm{~Hz}$, and the left and right frames are temporally divided [Woods and Helliwell 2011]. As an application of Virtual Reality (VR), Maneesh proposed two-viewer stereoscopic display using DLP [Agrawala et al. 1997], where four frames (LeftEye1, RightEye1, LeftEye2, RightEye2) were projected by time division.

\subsection{Glasses-free stereoscopic}

Glasses-free stereoscopic systems using DLP or LCD have been proposed as well. Jones proposed projecting binary patterns onto a rapidly spinning mirror, thereby enabling 360-degree views [Jones et al. 2007]. Cossairt projected patterns onto a moving slit by using a DLP projector [Cossairt et al. 2004][Cossairt et al. 2007]. Jones also proposed auto-multi-scopic 3D displays that allow a 
large number of viewers [Jones et al. 2015] by using 216 DLP projectors for light fields, where each light is reflected by a special material screen. Gordon proposed tensor displays, which synthesize an efficient light field using three LCD panels [Gordon et al. 2012].

\subsection{Image decomposition}

There are several techniques for the decomposition of multiple images. The eigen-decomposition of many facial images is used for face recognition, and is called "eigenface" [Turk and Pentland 1991]. Singular-value decomposition (SVD) of images or image filters is used for pattern recognition as well [Bie et al. 2005]. In these techniques, the original images are decomposed to basis images and basis functions, and a low-rank approximation is performed by truncating the number of bases. The basis images and basis function thus obtained are real values. Non-negative matrix factorization (NMF) can decompose a matrix into non-negative vectors [Lee and Seung 2000]. The decomposition model in this study is more special case: $0-1$ binary-basis images and positive continuous vectors for decomposition.

\subsection{Advantages of the proposed method}

Although our method is an extension of the conventional active shutter stereoscopic method, this enables a greater number of views, and reduces flicker and quantization errors in the same conditions as those for the conventional method. Furthermore, our method does not require special devices or many projectors. It does require a consumer DLP projector and slightly improved active shutter glasses. The projection size is easily controllable in the same manner as a standard projector, such as $5 \sim 100$ inches, and allows a large number of viewers.

\section{Imaging models}

In this section, we formulate the conventional and the proposed imaging models using a DLP projector.

\subsection{Formulation of DLP imaging model}

The DLP imaging model is defined as follows:

$$
f(x, y)=\sum_{n=1}^{N} B_{n}(x, y) L_{n} .
$$

Here, $B_{n}(x, y)$ represents the binary images of the DMDs and $L_{n}$ represents light intensities. $N$ indicates the number of the binary images.

For example, for an eight-bit grayscale image, the following normalized luminance coefficient $\mathbf{L}$ is used:

$$
\mathbf{L}=\frac{1}{255}[128,64,32,16,8,4,2,1]^{\mathrm{T}} .
$$

In this model, the projected image $0 \leq f(x, y) \leq 1$ can be presented.

\subsection{Multi-view display model with 0-1 active shutter}

Because of the rapidness of DLP projection, it is possible to use special glasses to display stereoscopic images in addition to views.

For example, in stereoscopic imaging, DLP alternately projects two images, the right and left views. The active shutter glasses, of which the right and left shutters can alternate between an on-off state, are controlled to achieve synchronization with the DLP projection. Finally, stereoscopic imaging can be realized. LCD or polarized glasses are well-known implementations of active shutters.

In this model, $M$ images $f^{i}$ can be represented as follows:

$$
f^{i}(x, y)=\sum_{n=1}^{N} B_{n}(x, y) r_{i}^{n} L_{n}, i=1,2, \cdots, M
$$

Here, $r_{i}^{n}=\{0,1\}$ indicates the state of the active shutter, where " 1 " is open and " 0 " is closed.

For example, in stereoscopic imaging with $M=2$, the following parameters are used:

$$
\left\{\begin{array}{l}
\mathbf{r}^{\mathbf{1}}=[1,1,1,1,0,0,0,0]^{T} \\
\mathbf{r}^{2}=[0,0,0,0,1,1,1,1]^{T}, \\
\mathbf{L}=\frac{1}{255}[128,64,32,16,128,64,32,16]^{T}
\end{array}\right.
$$

In this case, the right and left images $f^{1}$ and $f^{2}$ are presented by time division, and the depth of the images is reduced to four bits.

\subsection{Proposed method: Multi-view display model with analog shutter}

The technical problems associated with a conventional timedivision active shutter are as follows:

- $0-1$ shuttering causes flicker for human eyes because complete black patterns are projected. The flicker increases according to the number of time divisions $M$

- Quantization errors occur between input images and projected images because the number of binary patterns is divided by $M$.

We propose to solve the technical issues by relaxing the active shutter model as follows:

$$
\mathbf{r}^{\mathbf{i}}=\left[r_{1}^{i}, \cdots, r_{N}^{i}\right]^{\mathrm{T}}, 0 \leq r_{n}^{i} \leq 1 .
$$

This model means that the active shutter is not restricted to taking the 0-1 state, but uses continuous transmittance instead. Unlike the conventional 0-1 active shutter, the proposed model prevents the projection of extreme black patterns, and can reduce the flicker of the display. Furthermore, the quantization errors of imaging can be reduced because our model can use a larger number of binary patterns than the naive time-division system.

Here, we simplify the problem by maintaining the light intensity at the constant value of $L=1$ because the intensity of projected binary patterns is controllable by the continuous shutter $r_{i}^{n}$.

$$
f^{i}(x, y)=\sum_{n=1}^{N} B_{n}(x, y) r_{i}^{n} L, i=1,2, \cdots, M
$$

A summary of the proposed multi-view DLP imaging conversion follows.

Problem 1 Given multiple $M$ images as input $f^{i}(x, y)$, and number of binary patterns (image bits) $N$, determine optimal transmittance of active shutter glass $\mathbf{r}^{n}$ and binary patterns $B_{n}(x, y)$.

The technical issues associated with Problem 1, and our contributions in light of these, are as follows: 
1. We provide an efficient numerical solver for Problem 1. Problem 1 contains an NP-hard problem, which needs to be solved in realistic computational time.

2. We develop active shutter glasses with continuous transmittance. The proposed model assumes that active shutter glasses can take a state of continuous transmittance in the range $[0,1]$. Thus, such a device needs to be developed. We implement continuous transmittance by using PWM control for the LCD shutter.

\section{Binary continuous image decomposition}

We define the following least-squares error-based cost function between given input images $f^{i}$ and the reconstructed images by $B_{n}$ and $\mathbf{A}:=\left[\mathbf{r}^{1}, \cdots, \mathbf{r}^{M}\right]$ :

$$
\begin{aligned}
E(\mathbf{B}, \mathbf{A}) & :=\sum_{i=1}^{M} \sum_{x, y}^{W, H}\left\|f^{i}(x, y)-\sum_{n=1}^{N} B_{n}(x, y) r_{n}^{i}\right\|^{2} \\
& =\sum_{i=1}^{M} \sum_{x, y}^{W, H}\left\|f^{i}(x, y)-\mathbf{B}(x, y)^{\mathrm{T}} \mathbf{r}^{i}\right\|^{2}
\end{aligned}
$$

Here, $W$ and $H$ represent the size of the input image and $\mathbf{B}(x, y)=$ $\left[B_{1}(x, y), \cdots, B_{N}(x, y)\right]^{\mathrm{T}}$, respectively. Binary pattern $B_{n}$ and shutter transmittance $\mathbf{r}^{i}$ are obtained by minimizing $E$. However, due to the special constraints on the cost function $E, B_{n}(x, y)=$ $\{0,1\}$, and $0 \leq r_{n}^{i} \leq 1$, it is very difficult to achieve an exact minimization ${ }^{1}$ In other words, we need to decompose the input images to asymmetric form on a binary and continuous basis.

A possible approach to solving this difficulty is to decompose the problem into two steps: optimizing continuous variables $r^{i}$ and $0-1$ variables $B_{n}$. Thus, given the fixed $r^{i}$, the remainder of the problem becomes a 0-1 combinatorial optimization problem. We denote the cost function by $E(\mathbf{B} ; \mathbf{A})$.

In this study, we search for optimal shutter transmittance $\mathbf{r}^{i}$ by using a particle swarm optimization (PSO) algorithm. PSO is a powerful meta-heuristics search technique that does not require any assumptions about the problem, although PSO does not guarantee an optimal solution.

\subsection{PSO algorithm for shutter transmittance}

In the PSO algorithm, multiple particles $\mathbf{z}_{1}, \mathbf{z}_{2}, \cdots, \mathbf{z}_{m}$ are used, where $m$ is the number of particles. Each particle $z_{i}$ has velocity $\mathbf{v}_{i}=\left[v_{i}^{1}, \cdots, v_{i}^{M \times N}\right]^{T}$. In our model, an $M$-shutter transmittance $\mathbf{r}^{i}$ is assigned to $\mathbf{z} ; \mathbf{z}=\left\{r_{1}^{1}, \cdots, r_{N}^{1}, r_{1}^{2}, \cdots, r_{N}^{M}\right\}$. Then, the particles are updated as follows:

$$
\begin{aligned}
\mathbf{z}_{i}^{t+1}= & \mathbf{z}_{i}^{t}+\mathbf{v}_{i}^{t+1} \\
\mathbf{v}_{i}^{t+1}= & \omega_{0} \mathbf{v}_{i}^{t}+\omega_{1} \text { rand } \cdot\left(\mathbf{z}_{\text {pbest }, i}^{t}-\mathbf{z}_{i}^{t}\right) \\
& +\omega_{2} \text { rand } \cdot\left(\mathbf{z}_{\text {gbest }}^{t}-\mathbf{z}_{i}^{t}\right) .
\end{aligned}
$$

Here, rand is uniform random value with range $[0,1], \omega_{0}, \omega_{1}$, and $\omega_{2}$ are control parameters, and $t$ is a time step. $\mathbf{z}_{p b e s t, i}^{t}$ is the best solution for the particles given by:

$$
\mathbf{z}_{\text {pbest }, i}^{t}=\arg \min _{\mathbf{z}_{\mathbf{i}}^{\mathbf{t}^{\prime}, t^{\prime} \leq t}} E\left(\mathbf{B} ; \mathbf{z}_{\mathbf{i}}^{\mathbf{t}^{\prime}}\right)
$$

\footnotetext{
${ }^{1}$ For example, if there is no constraint on $B_{n}$ and $\mathbf{r}^{i}$, it is possible to use singular-value decomposition (SVD). However, we cannot use SVD in our model.
}

and $\mathbf{z}_{\text {gbest }, i}^{t}$ is the best known global swarm solution given by:

$$
\mathbf{z}_{\text {gbest }}^{t}=\arg \min _{i} E\left(\mathbf{B} ; \mathbf{z}_{\text {pbest }, i}^{t}\right)
$$

In the optimization process, the number of particles $m$ and the maximum iteration $t_{\max }$ are given, and the initial particles $\mathbf{z}_{i}^{0}$ and their velocities $\mathbf{z}_{i}^{0}$ are given randomly. Then, the particles are updated iteratively according to Eq. (9) until time step $t$ reaches $t_{\max }$. Iterations of the PSO can be used to gradually reduce cost function E.

\subsection{Binary pattern optimization}

The PSO algorithm requires the computation of $E(\mathbf{B} ; \mathbf{A})$. By giving $\mathbf{r}$, because cost function $E$ becomes independent of $(x, y)$, it is sufficient to optimize the following cost function at each pixel $(x, y)$.

$$
E(x, y)=\sum_{i=1}^{M}\left\|f^{i}-\mathbf{b}^{\mathrm{T}} \mathbf{r}^{i}\right\|^{2} .
$$

Here, $\mathbf{b}=\mathbf{B}(x, y)$ and $f^{i}=f(x, y)$. By using $\mathbf{A}$ and $\mathbf{f}=$ $\left[f^{1}, \cdots, f^{M}\right]^{\mathrm{T}}$,

$$
E(x, y)=\|\mathbf{f}\|^{2}-2 \mathbf{b}^{\mathrm{T}} \mathbf{A} \mathbf{f}+\|\mathbf{A b}\|^{2} .
$$

Because $\|\mathbf{f}\|^{2}$ is a constant value,

$$
\min _{\mathbf{b}} E(x, y) \Leftrightarrow \min _{\mathbf{b}}\left[\mathbf{b}^{\mathrm{T}} \mathbf{Q} \mathbf{b}+\mathbf{q}^{\mathrm{T}} \mathbf{b}\right]
$$

Here, $\mathbf{Q}=\mathbf{A} \mathbf{A}^{\mathrm{T}}, \mathbf{q}=-2 \mathbf{A f}$. Thus, we redefine the cost function as follows:

$$
E(\mathbf{b})=\mathbf{b}^{\mathrm{T}} \mathbf{Q} \mathbf{b}+\mathbf{q}^{\mathrm{T}} \mathbf{b} .
$$

This is a 0-1 quadratic programming problem. Because it is an NP-hard problem [Karp 1972], an efficient solver is required. Generally, to optimize the above integer programming problem, the branch-and-bound method can be used [Mori 2014]. In this method, by applying a linear relaxation, the constraint of the $0-1$ integer value is converted to a continuous value with range $[0,1]$ and the lower bound of the subproblem [Press et al. 1992]. Then, by using the lower bound, the search nodes can be cut efficiently.

On the contrary, in our model of Eq. (16), because it is a special case involving an integer quadratic programming problem, $\mathbf{b}=$ $\{0,1\}^{N}$ and $\mathbf{Q}$ is a symmetric matrix; with elements $Q_{i j}>0$ and $q_{i}<0$, the lower bound can be computed easily and efficiently without linear relaxation.

\subsubsection{Division into subproblem}

The branch-and-bound method performs a hierarchical tree search for nodes, and child nodes are cut by the lower bound of the pivot node. Thus, this search requires the computation of the cost of the pivot node and its lower bound.

The cost can be computed by dividing cost function $E$ at the divided binary vector $\mathbf{b}^{\mathrm{T}}=\left[\mathbf{b}_{1}^{\mathrm{T}}, \mathbf{b}_{2}^{\mathrm{T}}\right]$, where $\mathbf{b}_{1}$ and $\mathbf{b}_{2}$ are the $n_{1}$-bit and the $n_{2}$-bit vectors, respectively. When $\mathbf{b}_{1}$ is given and fixed, the remaining $2^{n_{2}}$-nodes from $\mathbf{b}_{2}=[0,0, \cdots, 0]^{\mathrm{T}}:=\mathbf{0}$ to $[1,1, \cdots, 1]^{\mathrm{T}}:=\mathbf{1}$ become the search nodes. We define the node with $\mathbf{b}_{2}=\mathbf{0},\left(n_{2} \geq 2\right)$ as the pivot node.

The divided cost function with fixed $\mathbf{b}_{1}$ is defined as follows:

$$
\begin{aligned}
E\left(\mathbf{b}_{\mathbf{2}} ; \mathbf{b}_{1}\right)= & \mathbf{b}_{1}^{\mathrm{T}} \mathbf{Q}_{11} \mathbf{b}_{1}+2 \mathbf{b}_{1}^{\mathrm{T}} \mathbf{Q}_{12} \mathbf{b}_{2} \\
& +\mathbf{b}_{2}^{\mathrm{T}} \mathbf{Q}_{22} \mathbf{b}_{2}+\mathbf{q}_{1}^{\mathrm{T}} \mathbf{b}_{1}+\mathbf{q}_{2}^{\mathrm{T}} \mathbf{b}_{2} \\
= & 2 \mathbf{b}_{1}^{\mathrm{T}} \mathbf{Q}_{12} \mathbf{b}_{2}+\mathbf{b}_{2}^{\mathrm{T}} \mathbf{Q}_{22} \mathbf{b}_{2}+\mathbf{q}_{2}^{\mathrm{T}} \mathbf{b}_{2}+c .
\end{aligned}
$$


Here,

$$
\mathbf{Q}=\left[\begin{array}{l:l}
\mathbf{Q}_{11} & \mathbf{Q}_{12} \\
\hdashline \mathbf{Q}_{12}^{\mathrm{T}} & \mathbf{Q}_{22}
\end{array}\right]
$$

and

$$
\mathbf{q}^{\mathrm{T}}=\left[\mathbf{q}_{1}^{\mathrm{T}}, \mathbf{q}_{2}^{\mathrm{T}}\right] .
$$

The divided matrices are $\mathbf{Q}_{11} \in \mathbf{R}^{n_{1} \times n_{1}}, \mathbf{Q}_{12} \in \mathbf{R}^{n_{1} \times n_{2}}$, and $\mathbf{Q}_{22} \in \mathbf{R}^{n_{2} \times n_{2}}$, and the vectors are $\mathbf{q}_{1} \in \mathbf{R}^{n_{1}}$ and $\mathbf{q}_{2} \in \mathbf{R}^{n_{2}}$. The constant value $c=E\left(\mathbf{0} ; \mathbf{b}_{1}\right)=\mathbf{b}_{1}^{\mathrm{T}} \mathbf{Q}_{11} \mathbf{b}_{1}+\mathbf{q}_{1}^{\mathrm{T}} \mathbf{b}_{1}$ can be obtained from Eq. (17).

\subsubsection{Lower bound analysis}

Following division into the subproblem, the given pivot node is calculated and, if the lower bound of the given pivot can be obtained, we can skip a search of the child nodes of the pivot according to the lower bound $\Delta E$ and the current minimum calculation $E_{\text {opt }}$.

We estimate the lower bound of the nodes from $\left[\mathbf{b}_{1}, 0, \cdots, 0\right]$ to $\left[\mathbf{b}_{1}, 1, \cdots, 1\right]$. Eq. (17) with given $\mathbf{b}_{1}$, and using conditions $Q_{i j} \geq$ 0 and $q_{i} \leq 0$, enables us to easily estimate an approximate lower bound as follows:

$$
\begin{aligned}
E\left(\mathbf{b}_{\mathbf{2}} ; \mathbf{b}_{\mathbf{1}}\right) & \geq \mathbf{q}_{\mathbf{2}}{ }^{\mathrm{T}} \mathbf{1}+E\left(\mathbf{0} ; \mathbf{b}_{\mathbf{1}}\right) \\
& =\Delta E_{1}+E\left(\mathbf{0} ; \mathbf{b}_{\mathbf{1}}\right) .
\end{aligned}
$$

Here, $\Delta E_{1}:=\mathbf{q}_{2}{ }^{\mathrm{T}} \mathbf{1}$. This equation means that after calculating the pivot node $E\left(\mathbf{0} ; \mathbf{b}_{1}\right)$, the approximate lower bound can be computed by adding $\Delta E_{1}$ without relying on $\mathbf{b}_{2}$.

We enhance the lower bound by transforming the cost function in Eq. (17) as follows:

$$
\begin{aligned}
E\left(\mathbf{b}_{\mathbf{2}} ; \mathbf{b}_{\mathbf{1}}\right) & \geq\left[2 \mathbf{b}_{1}^{\mathrm{T}} \mathbf{Q}_{12}+\mathbf{q}_{\mathbf{2}}^{\mathrm{T}}\right] \mathbf{b}_{\mathbf{2}}+c \\
& =\mathbf{u}^{\mathrm{T}} \mathbf{b}_{\mathbf{2}}+c \\
& \geq \mathbf{u}^{\prime \mathrm{T}} \mathbf{1}+c \\
& =\Delta E_{2}+E\left(\mathbf{0} ; \mathbf{b}_{\mathbf{1}}\right) .
\end{aligned}
$$

Here, $\Delta E_{2}:=\mathbf{u}^{\prime \mathrm{T}} \mathbf{1}$ and $u_{i}^{\prime}=\min \left(0, u_{i}\right)$. Furthermore, we can enhance the lower bound as follows:

$$
\begin{aligned}
E\left(\mathbf{b}_{2} ; \mathbf{b}_{1}\right)= & {\left[2 \mathbf{b}_{1}^{\mathrm{T}} \mathbf{Q}_{12}+\operatorname{diag}\left(\mathbf{Q}_{22}\right)^{\mathrm{T}}+\mathbf{q}_{2}^{\mathrm{T}}\right] \mathbf{b}_{2} } \\
& +\mathbf{b}_{2}^{\mathrm{T}} \hat{\mathbf{Q}}_{22} \mathbf{b}_{2}+c \\
= & \mathbf{m}^{\mathrm{T}} \mathbf{b}_{2}+\mathbf{b}_{2}^{\mathrm{T}} \hat{\mathbf{Q}}_{22} \mathbf{b}_{2}+c .
\end{aligned}
$$

Here, $\operatorname{diag}(\mathbf{A})$ denotes the vector the elements of which are given by the diagonal elements of matrix $\mathbf{A} ; \operatorname{diag}(\mathbf{A})=$ $\left[A_{11}, A_{22}, \cdots, A_{n n}\right]^{\mathrm{T}}$, and $\hat{\mathbf{A}}$ denotes the matrix where the diagonal elements of $\mathbf{A}$ are replaced by 0 and $\mathbf{m}:=2 \mathbf{b}_{\mathbf{1}}{ }^{\mathrm{T}} \mathbf{Q}_{\mathbf{1 2}}+$ $\operatorname{diag}\left(\mathbf{Q}_{\mathbf{2 2}}\right)^{\mathrm{T}}+\mathbf{q}_{\mathbf{2}}^{\mathrm{T}}$.

Because $Q_{i j} \geq 0$, the enhanced lower bound can be computed as follows:

$$
\begin{aligned}
E\left(\mathbf{b}_{2} ; \mathbf{b}_{1}\right) & \geq \mathbf{m}^{\mathrm{T}} \mathbf{b}_{2}+c \\
& \geq \mathbf{m}^{\prime \mathrm{T}} \mathbf{1}+E\left(\mathbf{0} ; \mathbf{b}_{1}\right) \\
& =\Delta E_{3}+E\left(\mathbf{0} ; \mathbf{b}_{1}\right)
\end{aligned}
$$

Here, $\Delta E_{3}:=\mathbf{m}^{\prime \mathrm{T}} \mathbf{1}$ and $m_{i}^{\prime}=\min \left(0, m_{i}\right)$. The procedure of binary pattern optimization is described in Algorithm 1.

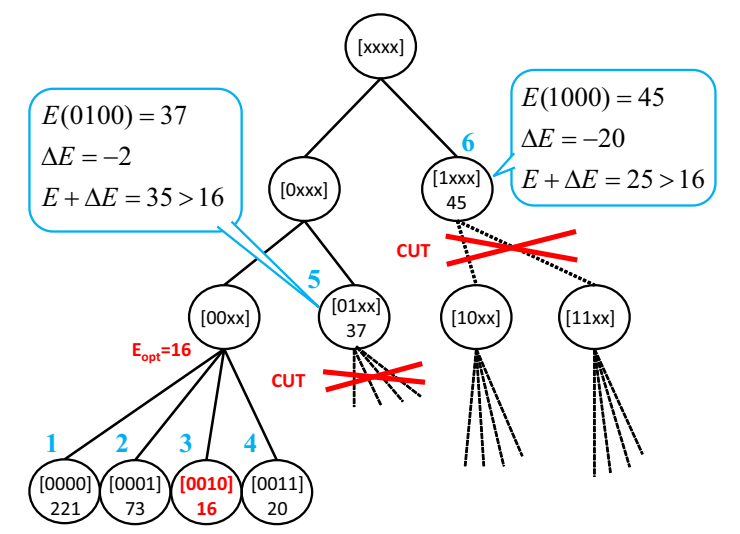

Figure 4: An example of search tree structure for a 0-1 quadratic programming problem. After computing the lower bound of the pivot node, the child nodes of the pivot node can be skipped for search.

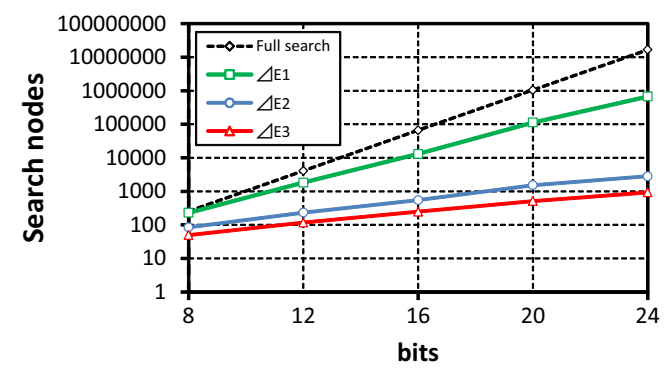

Figure 5: Number of search nodes for full search, cut by $\Delta E_{1}$, $\Delta E_{2}$, and $\Delta E_{3}$. The use of $\Delta E_{3}$ can significantly enhance search efficiency.

\subsubsection{Example of binary pattern optimization}

We provide a simple example of Algorithm 1 using Figure 4. The number of input images is $M=2$, and binary patterns $N=4$. The given parameters are as follows:

$$
\begin{aligned}
& \mathbf{A}=\left[\begin{array}{cccc}
2 & 10 & 4 & 4 \\
8 & 7 & 10 & 8
\end{array}\right]^{\mathrm{T}}, \\
& \mathbf{f}=[10,11]^{\mathrm{T}} .
\end{aligned}
$$

Until the first pivot node [0100] is reached, four nodes are calculated and temporary optimal cost $E_{o p t}=16$ is obtained. Then, at the pivot node [0100], the cost of the node and $\Delta E=-2$ is computed, and the lower bound $E+\Delta E=35$ is obtained. Because this bound is greater than $E_{\text {opt }}=16$, the child nodes of [0100] can be cut. In the same manner, the child nodes of the next pivot node [1000] can also be cut. Finally, an optimal bit [0010] and an optimal cost $E_{o p t}=16$ are obtained by calculating six nodes, although the full search requires $2^{4}=16$ nodes.

\subsection{Sampling calculation points}

The calculation of each particle using PSO necessitates the calculation of $E$ for all pixels $(x, y)$. However, the number of pixels is proportional to the image size $W \times H$, and the calculation is computationally intensive. Thus, we accelerate the computational time for calculation by only calculating a reduced number of pixels determined by $K$-random sampling. We denote the sampling pixels as evaluation points (EP). 

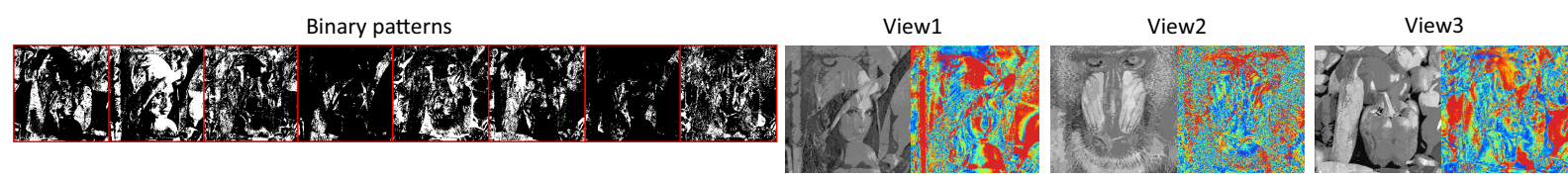

(a) Initial (Err = 13.66)
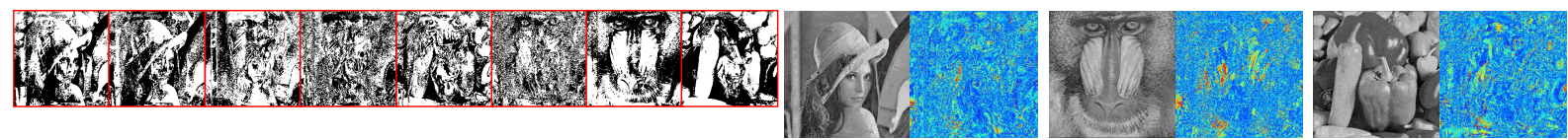

(b) 30 iterations $($ Err $=10.58)$
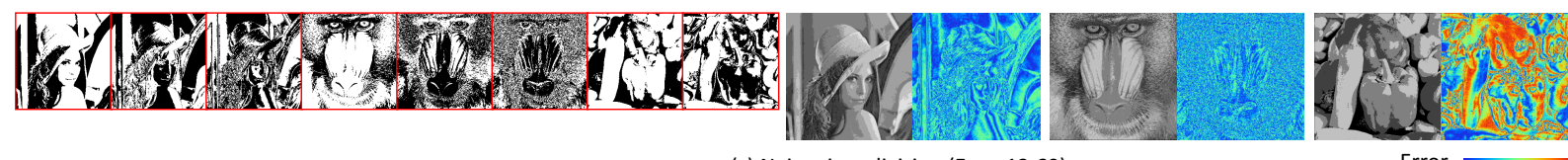

(c) Naive time division (Err $=13.60)$

Error

Figure 6: Simulation results for three-view images: Lena, Monkey, and Pepper. Results of the proposed method with (a) the initial solution, (b) after 30 iterations of the PSO algorithm. (c) Results of the naive time-division approach.

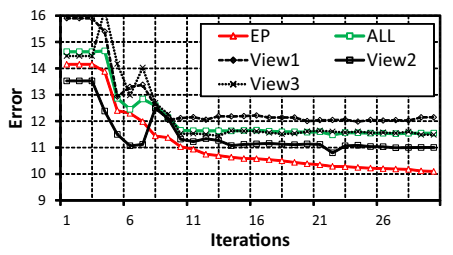

(a) $K=50$

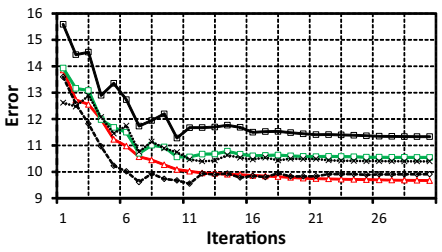

(b) $K=200$

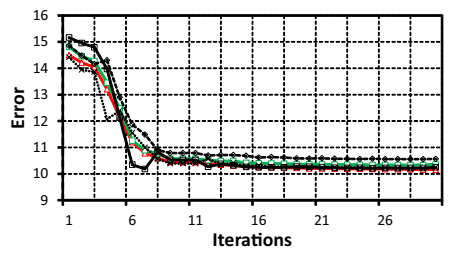

(c) $\mathrm{K}=1000$

Figure 7: Convergence of PSO algorithm with different values of random sampling parameter $K$. Increasing $K$ improves the estimated calculation for the entire set of pixels.

Table 1: Comparison of the number of search nodes.

\begin{tabular}{r|r|r|r|r}
\hline bit & Full & $\Delta E_{1}$ & $\Delta E_{2}$ & $\Delta E_{3}$ \\
\hline \hline 8 & 256 & 228.85 & 84.30 & 49.62 \\
12 & 4096 & 1820.76 & 231.91 & 116.70 \\
16 & 66536 & 13098.39 & 548.30 & 248.54 \\
20 & 1048576 & 113162.17 & 1527.36 & 512.30 \\
24 & 16777216 & 671769.65 & 2820.28 & 932.99 \\
\hline
\end{tabular}

\subsection{Effectiveness of each node cut}

We confirmed the effectiveness of branch-and-bound search by testing 100 numerical samples with varying numbers of bits, i.e., $N=8,12,16,20$, and 24 . Table 1 lists the results, which indicate the search nodes for each algorithm, and $(\cdot)$ denotes a factor of search nodes compared with a full search. In the table, "Full" indicates a full search and " $\Delta E_{1}, ", \Delta E_{2}, "$ and $" \Delta E_{3}$ " are the results of the branch-and-bound search with lower bound $\Delta E_{1}, \Delta E_{2}$ and $\Delta E_{3}$ in Eq. (21), Eq. (25), and Eq. (30), respectively. Furthermore, Figure 5 shows the results in Table 1 in graphical form.

The results in the table and the graph enable the number of search nodes to be efficiently reduced. In particular, the node cut using $\Delta E_{3}$ is very powerful, and the use of 20 bits allows 2,046 factors to be obtained. Here, the obtained $\min E$ and $\mathbf{b}$ using the branchand-bound search are equivalent to the results of a full search.

\section{Simulation results}

We applied our algorithm to images and evaluated the root-meansquared (RMS) errors compared with the ground truth. In this sim- ulation, two sets of images were calculated: those with three views and with five views. In this evaluation, RMS error was defined as follows:

$$
\operatorname{RMS}:=\sqrt{\frac{1}{M \cdot W \cdot H}\left\|f^{i}(x, y)-\hat{f}^{i}(x, y)\right\|^{2}} .
$$

Here, $\hat{f}$ is the reconstructed image. Code for the optimization and the simulation was written in $\mathrm{C}++$ on a $\mathrm{PC}$ spec core-i7, $3.4 \mathrm{GHz}$, and $8 \mathrm{~GB}$ of DRAM.

\subsection{Three views}

The result obtained with the three views is shown in Figure 6. The images used were Lena, Monkey, and Pepper, and were obtained from the USC-SIPI image database. The size of each image was $256 \times 256$, and each image was converted into eight-bit grayscale. Our algorithm was used to decompose the three images $(M=3)$ into eight binary patterns $(N=8)$. The left and right parts of Figure 6 show the decomposed binary patterns and the reconstructed images, respectively. The pseudo-colored images show the errors between the reconstructed image and the ground truth. Figures 6 (a)(b) indicate the results of the initial solution and after 30 iterations, respectively. As shown in the figure, the reconstruction errors were reduced by increasing the number of iterations. Figure 6 (c) shows the results of the naive time-division approach. In this case, $\mathbf{r}^{1}=[1,1,1,0,0,0,0,0]^{\mathrm{T}}, \mathbf{r}^{2}=[0,0,0,1,1,1,0,0]^{\mathrm{T}}$, and $\mathbf{r}^{3}=[0,0,0,0,0,0,1,1]^{\mathrm{T}}$ were used, which means that view1 and view2 were three-bit depth images, whereas view3 was a two-bit image. Because of the low-bit representation, the image of view3 had large quantization errors. The average RMS error of the naive method was 13.60 , and this was improved to 10.58 by using the proposed method. 

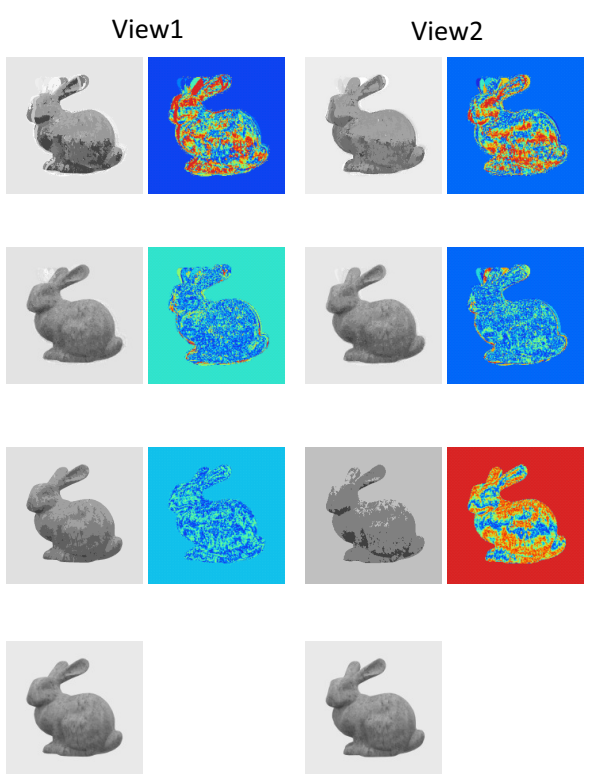
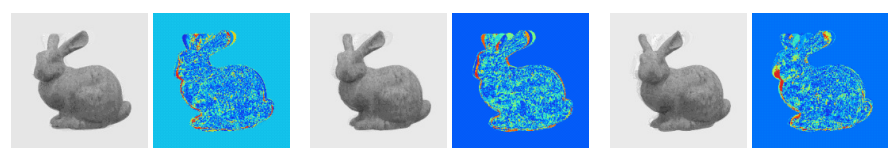

(b) 30 iterations
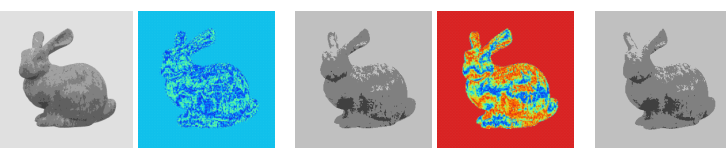

(c) Naive
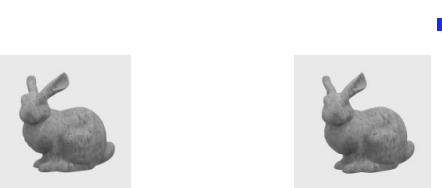

View5

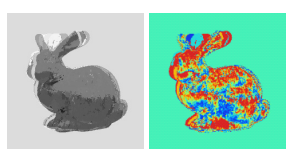

(d) Ground truth

Figure 8: Simulation results for five-view images: (a) Representation using the proposed method. (b) After 30 iterations. (c) Naive timedivision method. (d) Ground truth. The naive method indicates a large quantization error because this can use only two or three bits per view.

Table 2: Computation time for different values of $K$.

\begin{tabular}{l|r|r|r|r}
\hline $\mathrm{K}$ & time/iteration[ms] & EP & All & $\mid$ EP-All $\mid$ \\
\hline \hline 50 & 175 & 10.10 & 11.55 & 1.45 \\
200 & 789 & 9.67 & 10.55 & 0.88 \\
500 & 1729 & 9.84 & 10.20 & 0.36 \\
1000 & 4039 & 10.16 & 10.35 & 0.20 \\
2000 & 7020 & 10.32 & 10.46 & 0.15 \\
\hline
\end{tabular}

We confirmed the effectiveness of the sampling of evaluation points by using different parameters for the evaluation: namely $K=$ 50, 200,500, 1000, and 2000. Table 2 lists the results, where "EP" indicates RMS errors at the sampled evaluation points and "All" indicates those across all pixels. The results in the table indicate that a smaller value of $K$ improved the required computation time; however, the difference between "EP" and "All" increased. Figure 7 shows the convergence of the PSO algorithm with varying parameter values: $K=50,200$, and 1000 . As shown in the graph, PSO error converges early in the iterations.

Although three completely different images were used, binary continuous decomposition was successfully achieved.

\subsection{Five views}

The results obtained for five views are shown in Figure 8. Unlike in the case of three views, similar images were used in this case. The Stanford bunny was rendered at $-20,-10,0,+10$, and +20 degrees of rotation about the y-axis. The parameters $M=5, N=$ 12 bits, and $K=1000$ were used. As shown in the figure, the proposed algorithm reduced quantization errors for five images, and the RMS was reduced to 5.9. By comparison, the RMS obtained by the naive method was 15.7. Naive time division was used to divide the five-view images into two-bit or two-bit images, respectively.

\subsection{Spatio-temporal frequency analysis}

To evaluate the flicker in sub-frame sequence that is visible to the human eye, we compute the spatio-temporal frequency distribution of the $i$-th view as follows:

$F^{i}\left(f_{x}, f_{y}, f_{t}\right)=\sum_{x=0}^{W-1} \sum_{y=0}^{H-1} \sum_{t=0}^{N-1} B_{t}(x, y) r_{t}^{i} e^{-j 2 \pi\left[\frac{f_{x} \cdot x}{W}+\frac{f_{y} \cdot y}{H}+\frac{f_{t} \cdot t}{N}\right]}$.

$f_{x}$ and $f_{y}$ indicate a spatial frequency, and $f_{t}$ indicates a temporal frequency. A higher power spectrum at the high temporal frequency causes a stronger flicker because it implies rapid and large changes in the intensity of light.

In this evaluation, the energy distribution was separated into three spatial frequencies-"high spatial frequency," "mid-spatial frequency," and "low spatial frequency"- using three bandpass filters shown in Figure 9 (a). Here, we used a simple linear bandpass filter. For example, spatial low-frequency component $F_{L o w}^{i}$ is computed by follows:

$$
F_{\text {Low }}^{i}\left(f_{x}, f_{y}, f_{t}\right)=F^{i}\left(f_{x}, f_{y}, f_{t}\right) \cdot f_{\text {Low }}(r) .
$$

Here, the spatial radius is defined as $r=\sqrt{f_{x}^{2}+f_{y}^{2}}$.

Figure 9 (b) shows an example of the 3D spatio-temporal power spectrum obtained using Eq. (34). Then, the following three sequences were analyzed and compared, as shown in Figure. 10.

(a) Naive time division Once non-black binary patterns are projected in succession, black frames are projected for a long time.

(b) Conventional time division To avoid a long time black frame, non-black binary patterns are inserted at regular time intervals.

(c) Proposed All frames are non-black binary patterns. 


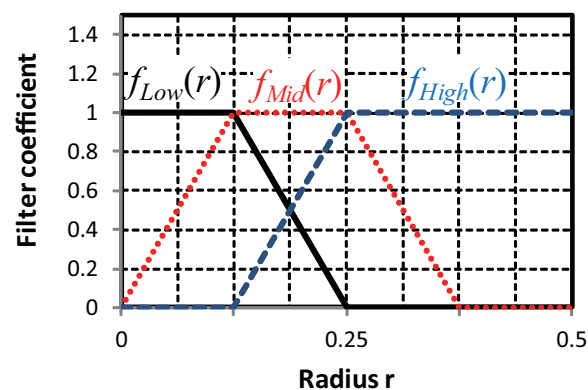

(a) Three-bandpass filters

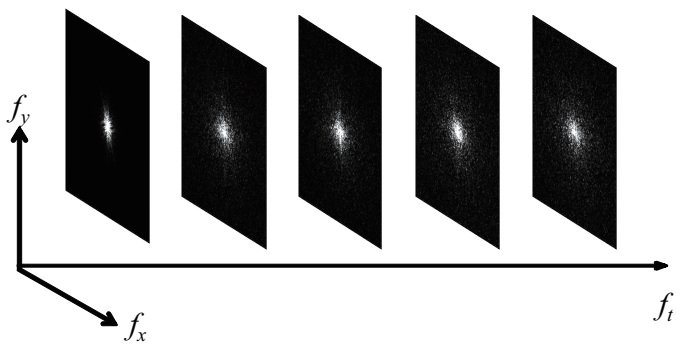

(b) Spatio-Temporal spectral

Figure 9: Spatio-temporal discrete Fourier transformation. (a) Three bandpass filters for low, mid, and high frequency. (b) Image sequence was converted to the spatio-temporal domain, and power spectra were computed for frequency analysis.

The spatio-temporal power spectra for three views and five views are shown in Figure 11 and Figure 12, respectively. In this evaluation, the average values of each spatial power spectrum of $F_{L o w}^{i}, F_{M i d}^{i}$ and $F_{H i g h}^{i}$ were computed. "Naiv-Low" in this figure shows the average power spectrum of $F_{L o w}^{i}$ using the naive time division. "Conv-Low" and "Prop-Low" show the average power spectrum using the conventional time division and the proposed method, respectively. From the figure, a conventional time-division method increases the high-temporal frequency component than the naive method. On the contrary, the proposed method can reduce a greater amount of energy at high temporal frequencies than other methods. Most of the energy is concentrated at low spatial and temporal frequencies in the three methods. Figure 12 (c) shows midand high-spatial frequencies, which indicate the same trend as a low-spatial frequency.

\section{Implementation}

Our model assumes that the LCD shutter can accept continuous intermediate transmittance. We implemented a gray-level LCD shutter by using PWM to control it. The state of the LCD shutter was controlled by the following duty rate $D$ :

$$
D:=\frac{T_{o n}}{T}, T=T_{o n}+T_{o f f}
$$

Here, $T_{o n}$ and $T_{\text {off }}$ represent the closing and opening times of the LCD shutter. The state of the LCD shutter is controlled at regular time intervals $T$.

Figure 13 shows the circuit that we developed for LCD control as well as its schematic representation. PWM signals were generated by an eight-bit digital-to-analog converter (DAC) and the output
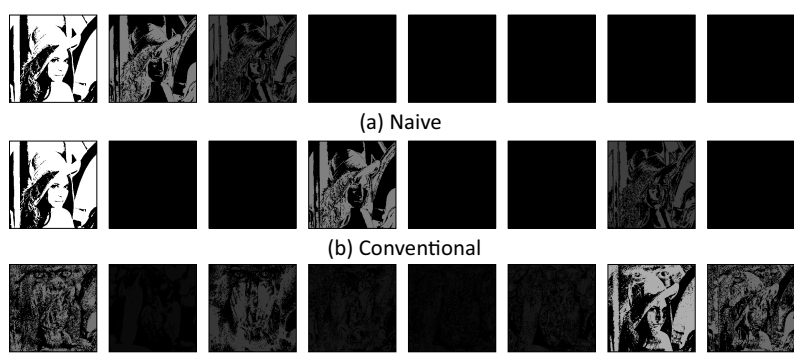

(c) Proposed

Figure 10: Three compared time sequences. (a) Naive time division. (b) Conventional time division. Binary patterns are projected alternately. (c) The proposed frame sequence.

current from the DAC was converted to voltage (I-V transform) using an operational amplifier (OpAmp). The peak-to-peak voltage of the OpAmp was controlled by a feedback resistor $R_{f}$. The DAC was controlled by a digital signal processor (DSP) microcontroller and enabled the DSP to generate a synchronization signal for the projector. The LCD panel was controlled alternately by positive and negative voltages because the voltage bias causes damage to the LCD. The alternate voltage maintained the bias close to $0 \mathrm{~V}$.

In our implementation, PIC16F1459 with a $4 \mathrm{MHz}$ Xtal oscillator, DAC0800 with parallel eight bits, and NJM4558D were used for the DSP, DAC, and OpAmp, respectively. The LCD shutter was an RV-3DGBT2B equipped with cost-effective consumer 3D active glasses with an STN-LCD panel. A voltage of $\pm 12 \mathrm{~V}$ was used for the LCD shutter.

\subsection{Response and linearity of LCD shutter}

We confirmed the effectiveness of the LCD shutter by developing the measurement circuit shown in Figure 14. This instrument contained LEDs under the LCD shutter, and the light they emitted was diffused by an acrylic diffuser. Following diffusion, the luminance of the diffused light was captured by a photo-diode positioned above the LCD shutter.

Figure 15(a) shows an overview of the control signal and LCD transmittance. Figure 15(c) shows an actual measured control PWM (red line) and the response signal (blue line) from the photodiode. In this case, $T=8.5 \mathrm{~ms}$ and $D=0.5$ were used. As shown in the figure, the time required to close and open the shutter was approximately $2 \mathrm{~ms}$. Because the LCD shutter behaves similarly to a capacitor, LCD discharge takes time. The latency was too low, and this was highly problematic for our system. In general, the response time of the LCD shutter was slow.

However, it is possible to accelerate LCD response by using a PWM signal with a short width for closing. Figure 15(b) shows an overview of short-time PWM control. By shorting a charge time to LCD, the time taken to discharge was also reduced. Figure $15(\mathrm{~d})$ shows the actual measured signals, where $T=500 \mu \mathrm{s}$ and $D=0.5$ were used. Although the LCD did not reach a static state, the response signal was periodic and the repeatability of the signals was high. As a result, an intermediate transmittance could be obtained.

We confirmed the relationship between duty rate $D$ and the transmittance of the LCD shutter by measuring the response of the photo-diode when changing from $D=0.0$ to $D=1.0$. Figure 14 (b) shows the result. A hundred samples were measured for each duty rate, and the average values and error bars are plotted. As shown in the figure, the linearity obtained was acceptable, and the standard deviation was approximately $0.5 \%$. Because the linearity 


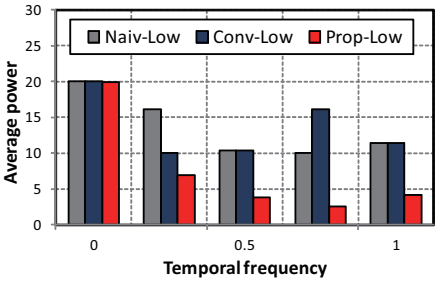

(a) View 1

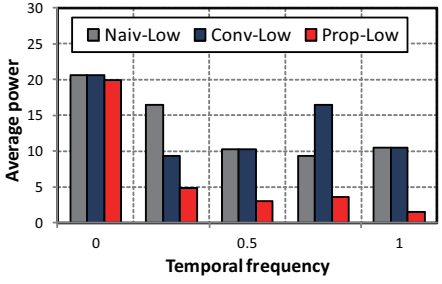

(b) View2

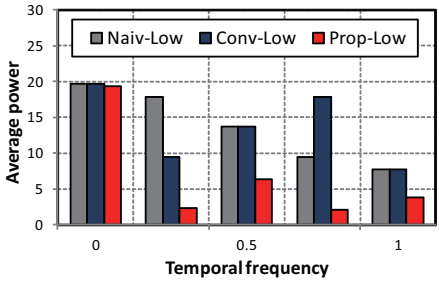

(c) View3

Figure 11: Spatio-temporal power spectra for three views. The spectra of the naive and conventional time division indicate large energy at high temporal frequency. The spectra of the proposed method yields less energy at a high temporal frequency.

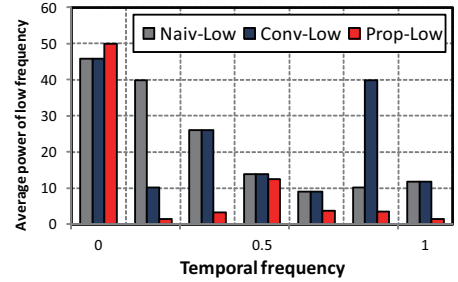

(a) View 1

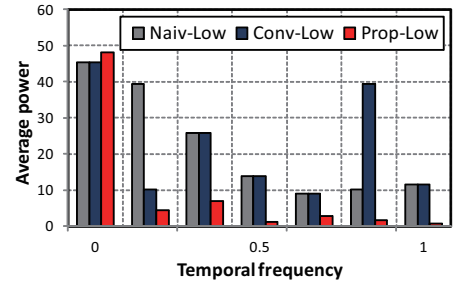

(b) View2

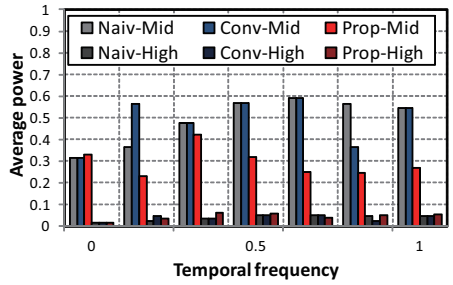

(c) Mid and High components

Figure 12: Spatio-temporal power spectra for five views. The same trend as in the three views can be obtained, and the mid-and high-value components indicate the same trend as with low-value components.

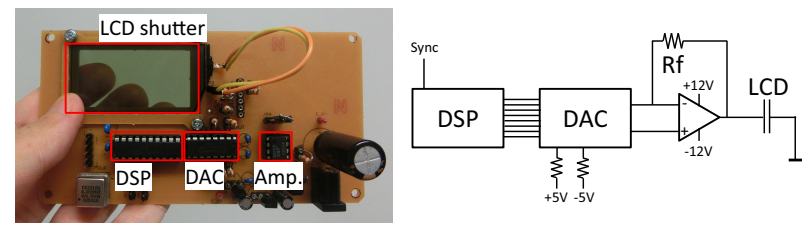

Figure 13: Prototype LCD shutter and schematic. The transmittance of the LCD shutter was controlled by the PWM signal generated by the DSP.

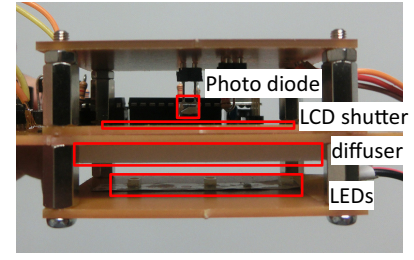

(a) Instruments

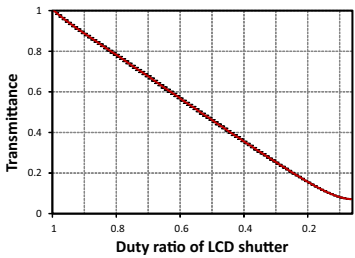

(b) Measured transmittance
Figure 14: (a) The developed measurement instrument. Light from the LEDs was transmitted through the LCD shutter, the transmittance of which was measured by the photo-diode. (b) Measured transmittance. Satisfactorily linearity between the duty rate of $P W M$ and transmittance was obtained.

was sufficient to represent a grayscale image, the optimized transmittance parameter $\mathbf{r}^{i}$ was available for use without calibration.

Note that this acceleration can improve the response time of the LCD shutter. However, the contrast of the LCD decreased as shown in Figure 15(b). Thus, there is a trade-off between response time and contrast. One possible, simple solution to this problem would be to improve contrast by increasing the brightness of the LEDs of the DLP projector.

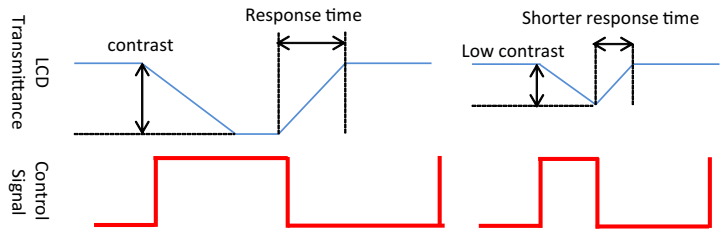

(a) Conventional PWM

(b) Short-time PWM

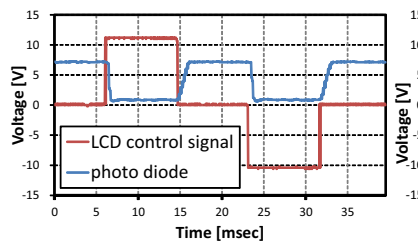

(c) PWM control T $=8.5 \mathrm{~ms}$

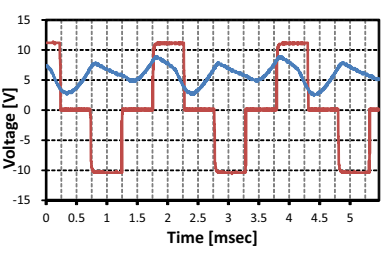

(d) PWM control T $=0.5 \mathrm{~ms}$
Figure 15: $P W M$ control signals and photo-diode signals response. (a)(c) Conventional PWM control. $\quad(b)(d)$ Improved short-time PWM control.

\subsection{Results and discussion}

By using optimized images $\mathbf{B}_{n}$ and transmittance parameters $\mathbf{r}^{i}$, we projected patterns onto a screen and captured the projected image with a CMOS camera. Figure 16 shows the image capturing system that we developed. A DLP projector, acrylic screen, LCD shutter, and CMOS camera were fixed to aluminum frames. Rearprojection film was placed on the acrylic screen, and the image projected by the DLP projector was captured from the back by the CMOS camera equipped with an LCD shutter. The DLP projector was a LightCrafter with $654 \times 608$ DMDs, and the CMOS camera was a Point Grey Flea3 with $1280 \times 960$ pixels. The time required to change the DMD and the LCD shutter was $T=1 \mathrm{~ms}$, and the frame rate was $1000 \mathrm{~Hz}$. We prevented the image captured by the rolling shutter from flickering by synchronizing the shutter trigger of the CMOS camera with the DLP projection and setting the exposure time to $80 \mathrm{~ms}$. 

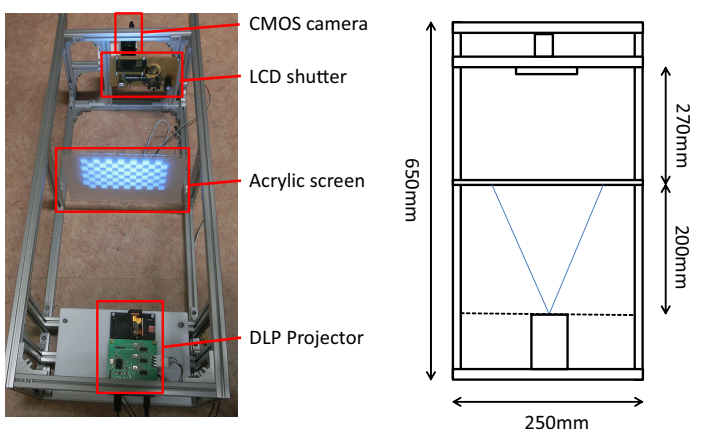

Figure 16: The developed DLP projector and LCD shutter system. The projected image was captured by a CMOS camera with an LCD shutter.
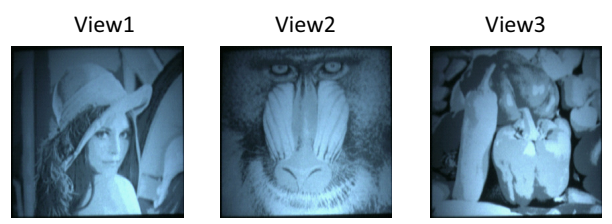

(a) Naive
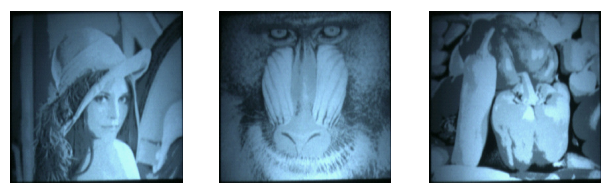

(b) Conventional
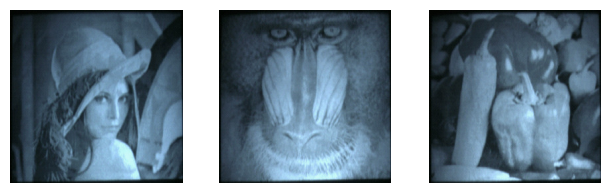

(c) Proposed

Figure 17: Results of actual captured images for three views by CMOS camera. In the conventional method, view1 and view 2 used three bits, and view3 used two bits for representation. This low-bit representation caused a large quantization error.

Figure 17 and Figure 18 show the captured images for each view. A two-image set similar to the simulation in the previous section was used. Figure 17 and Figure 18 (a)(b) show the result of the naive time-division method and the conventional time-division method, and Figure 17 and Figure 18 (c) show the result obtained by using the proposed method. As shown in the figure, the projected image was nearly identical to the simulation result. Note that the proposed method provided unique cross-talk artifacts as shown in Figure 19. This cross-talk was confirmed in both the simulation and the projected images. In particular, there was a large amount of cross-talk around the edges of objects. Figure 20 shows the sequential binary patterns for five views. Figures (a), (b), and (c) show the naive, conventional, and the proposed binary patterns. As shown in the figure, the conventional binary patterns contain 9 "black" frames; on the contrary, the proposed binary patterns can avoid these extreme black frames.

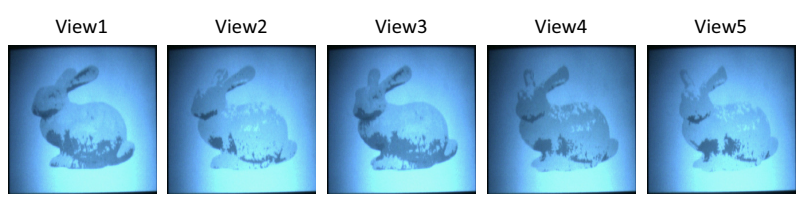

(a) Naive
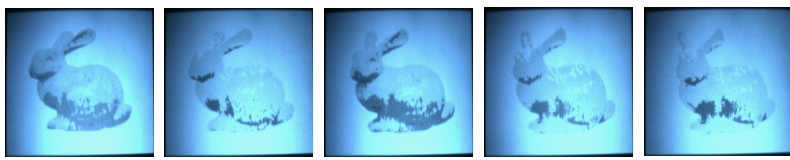

(b) Conventional
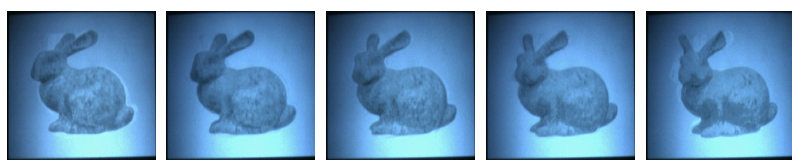

(c) Proposed

Figure 18: Results of actual captured images for five views by CMOS camera. Because there were many views for time division, the naive and conventional methods could use only two or three bits per view.

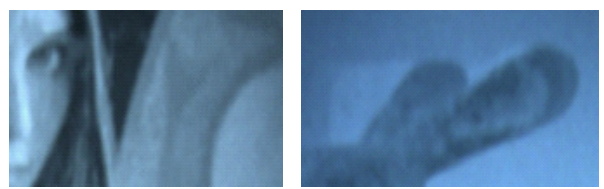

(a) Projected images

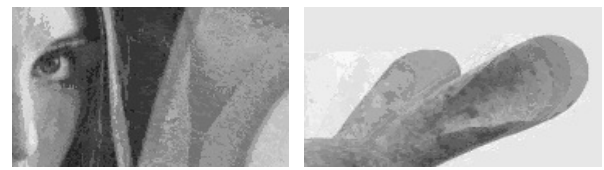

(b) Simulated images

Figure 19: The proposed method provided unique cross-talk. The artifact appeared in both the simulation and the projected image.

\subsection{Limitations}

\subsubsection{Maximum number of views}

Because the time required to tilt the DMDs was approximately $250 \mu \mathrm{s}$ at best, the limit of the frame rate for each binary pattern was $4000 \mathrm{~Hz}$. In case of a $60 \mathrm{~Hz}$ movie, $4000 / 60 \approx 66$ bit was limited and, for full-color RGB imaging, 22 bits per color channel was the limitation. Because a 12-bit binary pattern can represent five-view images, the maximum number of views was estimated to be approximately nine views with $60 \mathrm{~Hz}$. However, in the case of $30 \mathrm{~Hz}$ and monocolor imaging, $9 \times 6=54$ views were realized.

\subsubsection{Computation time}

The computation time required for the optimization of three-view and eight-bit images was approximately 20 seconds, whereas the time required for five-view and 12-bit images was approximately one minute. The results in Table 1 indicate that each time the number of bits increased by four, the computation time increased by a factor of $2 \sim 3$. Thus, the use of 22 bits would enable optimization in a realistic amount of time. 


\subsubsection{LCD shutter speed}

In this study, LCD shutter speed was accelerated by using a short PWM signal. However, it caused the contrast of the LCD panel to decrease. Shortening the time to less than $1 \mathrm{~ms}$ was expected to be difficult in light of the limitation presented by the LCD shutter. However, in recent years, the micro-electro-mechanical systems (MEMS) active shutter has been developed [Wu and Steyn 2013], and is expected to solve this problem because the shutter speed equals the tilting speed of the DMD.

\section{Conclusion and future work}

We extended the conventional $0-1$ active stereoscopic shutter to a continuous transmittance active shutter system. By introducing this extension, the DLP imaging model was re-formulated and a new problem-binary continuous image decomposition-was proposed. We presented an efficient numerical solver for the problem, and used both a simulation and an actual imaging system to prove that our model could represent multi-view images more accurately than the conventional time-division representation. In this study, although we established the feasibility and fundamentals of multiview display, there is room for developments in future research as follows:

VR appliactions We need to improve active shutter glasses to attain a more reduced size, a more rapid response time, higher contrast, and to add position tracking of the glasses by using IR markers [Rashid 1980]. Extension to full RGB color can be realized using multiple projectors. Halftone and dithering techniques can reduce the quantization error [Floyd and Steinberg 1975]. Furthermore, psychological evaluation involving human subjects is required.

Optimization Although the proposed PSO can provide a suboptimal solution, it does not guarantee a global optimal solution. To improve the solution and accelerate optimization time, a more efficient algorithm is needed. Furthermore, the method used to calculate the sample evaluation point (EP) can be improved.

Extension to LCD projector The proposed idea is not limited to binary DLP projectors, and can be applied to grayscale LCD projectors. In this extension, "multi-valued continuous decomposition" will be formulated.

\section{Acknowledgements}

Gou Koutaki thanks Dr. Katsuyuki Nakamura for providing online latex environment. Although the earthquake in Kumamoto at 14 April destroyed author's PC and laboratory, he was able to finish the paper using this tool.

\section{References}

Agrawala, M., Beers, A. C., McDowall, I., Fröhlich, B., Bolas, M., And Hanrahan, P. 1997. The two-user responsive workbench: Support for collaboration through individual views of a shared space. In SIGGRAPH '97, 327-332.

Bie, T. D., Cristianini, N., And Rosipal, R. 2005. Eigenproblems in pattern recognition. Handbook of Geometric Computing.

Cossairt, O., Travis, A. R. L., Moller, C., And Benton, S. A., 2004. Novel view sequential display based on dmd technology.

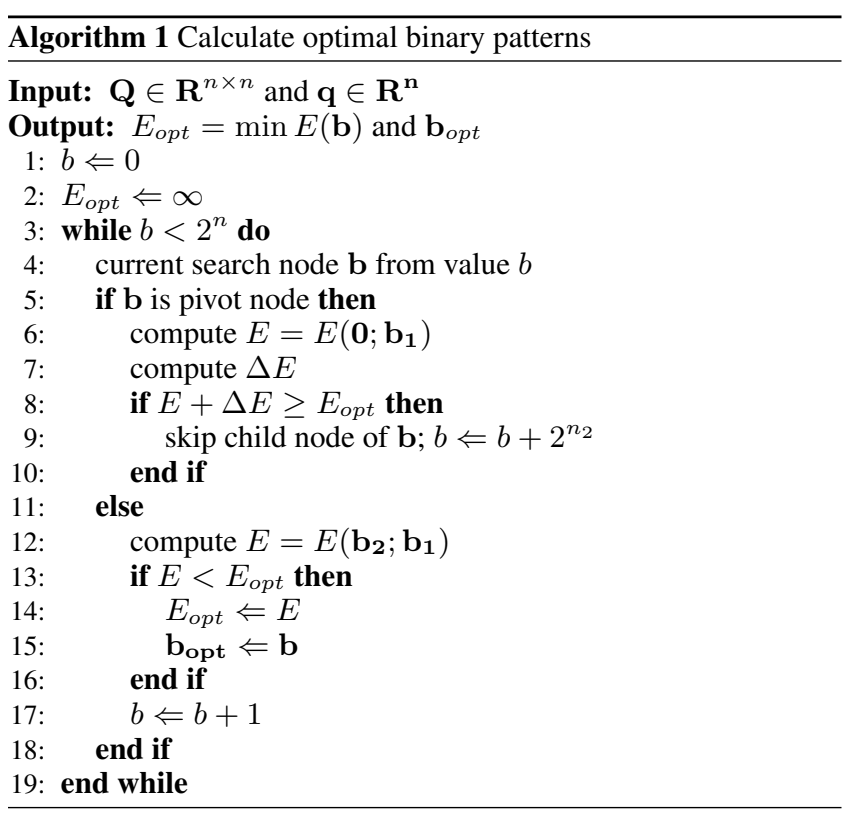

Cossairt, O., Napoli, J., Hill, S. L., Dorval, R. K., AND FAVALORA, G. E. 2007. Occlusion-capable multiview volumetric three-dimensional display. Applied Optics 46, 8, 1244-1250.

Floyd, R., AND STEINBERG, L. 1975. An adaptive algorithm for spatial gray scale. In International Symposium Digest of Technical Papers, Society for Information Displays, vol. 4, 36-37.

Gordon, W., Douglas, L., Matthew, H., and Ramesh, R. 2012. Tensor Displays: Compressive Light Field Synthesis using Multilayer Displays with Directional Backlighting. ACM Trans. Graph. (Proc. SIGGRAPH) 31, 4, 1-11.

Gove, R. J. 1994. DMD display systems: The impact of an alldigital display. In Information Display International Symposium, $1-12$.

Hammond, L., 1922. Stereoscopic motion picture, Mar. 2. US Patent $1,435,520$.

HORNBECK, L. J. 1995. Digital light processing and MEMS: Timely convergence for a bright future. In SPIE Micromachining and Microfabrication, 27-40.

Jones, A., McDowall, I., Yamada, H., Bolas, M., And DEBEVEC, P. 2007. Rendering for an interactive $360^{\circ}$ light field display. ACM Trans. Graph. 26, 3.

Jones, A., Unger, J., Nagano, K., Busch, J., Yu, X., Peng, H.-Y., Alexander, O., Bolas, M., And Debevec, P. 2015. An automultiscopic projector array for interactive digital humans. In ACM SIGGRAPH 2015 Emerging Technologies, SIGGRAPH ' 15, 6:1-6:1.

KARP, R. M. 1972. Reducibility among Combinatorial Problems. Plenum Press.

LeE, D. D., And Seung, H. S. 2000. Algorithms for nonnegative matrix factorization. In NIPS, MIT Press, 556-562.

MORI, K. 2014. Tractable global optimization algorithms for small boolean quadratic programming problems without multiplications and floating-point operations. Transactions of the Institute of Systems, Control and Information Engineers, 254-258. 


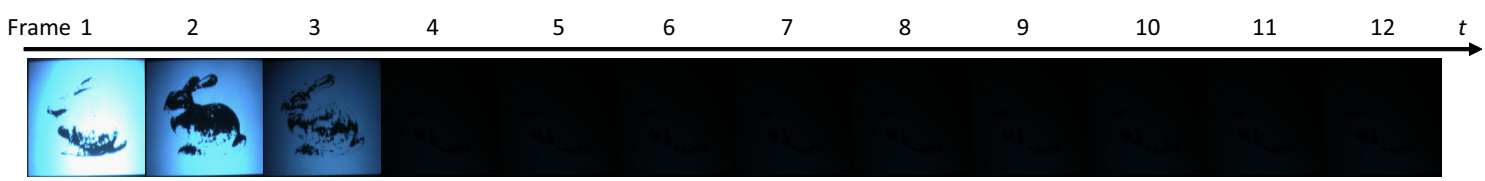

(a) Naive

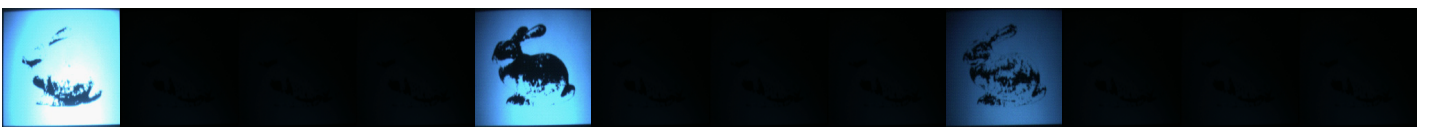

(b) Conventional

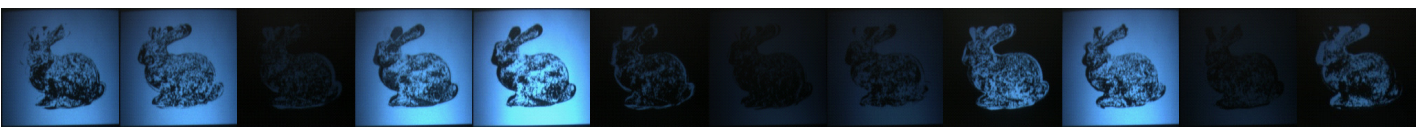

(c) Proposed

Figure 20: Projected binary patterns. (a) Patterns of the naive time-division method. (b) Conventional time-division method. (c) Proposed method. The naive and conventional methods include nine "black" frames. This causes strong flicker for the human eye.

Peddie, J. 2013. The History of Visual Magic in Computers: How Beautiful Images Are Made in CAD, 3D, VR and AR. Springer Publishing Company, Incorporated.

Press, W. H., Teukolsky, S. A., Vetterling, W. T., And FlanNeRY, B. P. 1992. Numerical Recipes in C (2Nd Ed.): The Art of Scientific Computing. Cambridge University Press.

RASHID, R. 1980. Toward a system for the interpretation of moving light display. IEEE trans. on Pattern Analysis and Machine Intelligence, 574-581.

Turk, M., AND Pentland, A. 1991. Face recognition using eigenfaces. In Proceedings of Computer Vision and Pattern Recognition, 586-591.

Urey, H., Chellappan, K. V., Erden, E., and Surman, P. 2011. State of the art in stereoscopic and autostereoscopic displays. Proceedings of the IEEE 99, 540 - 555.

Woods, A., AND Helliwell, J. 2011. White paper: A survey of $3 \mathrm{~d}$ sync ir protocols.

Wu, J. H., AND STEYN, J. L., 2013. Reliability studies on mems shutters and displays. 